\title{
GMR
}

\section{Expression and clinical significance of LRRC4 in benign and malignant nasopharyngeal diseases}

\author{
X.-J. Zhou', Y. Wang ${ }^{2}$, L.-J. Zhang ${ }^{3}$ and J.-H. Chen ${ }^{4}$ \\ ${ }^{1}$ Otolaryngology Department, Zhongshan Hospital of Chinese Medicine, \\ Zhongshan, China \\ 2Pathology Department, Zhongshan Hospital of Chinese Medicine, Zhongshan, China \\ ${ }^{3}$ Otolaryngology Department, Zhongshan Hospital of Chinese Medicine, \\ Zhongshan, China \\ ${ }^{4}$ Clinical Laboratory Department, Zhongshan Hospital of Chinese Medicine, \\ Zhongshan, China \\ Corresponding author: X.-J. Zhou \\ E-mail: zhxjls@yeah.net \\ Genet. Mol. Res. 14 (4): 16403-16409 (2015) \\ Received July 2, 2015 \\ Accepted October 6, 2015 \\ Published December 9, 2015 \\ DOI http://dx.doi.org/10.4238/2015.December.9.9
}

ABSTRACT. The aim of this study was to investigate the expression of LRRC4 in nasopharyngeal carcinomas, nasopharyngeal precancerous lesions, and nasopharyngitis as well as the clinical significance of LRRC4. Fifty patients with nasopharyngeal carcinoma were selected as study subjects; 28 patients with chronic nasopharyngitis and 22 patients with nasopharyngeal precancerous lesions served as controls. Immunohistochemical analysis was used to study protein expression of LRRC4; the relation between LRRC4 expression and the clinical stage and histopathological features of nasopharyngeal carcinoma was also analyzed. The LRRC4 expression manifested itself as yellow staining in the cytoplasm or nucleus. LRRC4 was strongly expressed in nasopharyngeal epithelial tissues of patients with chronic nasopharyngitis and in nasopharyngeal precancerous lesions; the rates of positive results were 82.1 and $81.8 \%$, 
respectively. LRRC4 was weakly expressed in nasopharyngeal carcinoma tissues, at a rate of $10 \%$ positive results $(P<0.001)$; there was no significant difference in the expression of LRRC4 among different clinical stages and pathological grades. Therefore, disappearance of LRRC4 expression is a major feature of nasopharyngeal carcinoma.

Key words: LRRC4; Nasopharyngeal carcinoma; Nasopharyngitis; Nasopharyngeal precancerous lesion

\section{INTRODUCTION}

LRRC4 (leucine-rich repeats containing 4), a new member of the leucine-rich repeat (LRR) superfamily, is a gene involved in the development of and axonal growth in the nervous system; LRRC4 is specifically expressed in healthy brain tissue and is downregulated or absent in a variety of primary brain tumors (Wang et al., 2002; Wu et al., 2006). Nasopharyngeal cancer is a primary malignant tumor in the nasopharynx (which is adjacent to the brain) and can easily invade and metastasize to the skull base; our previous studies have shown that, in contrast to nasopharyngitis, LRRC4 is methylated in nasopharyngeal carcinoma (Zhou et al., 2013). Later, studies involving immunofluorescence quantitative PCR also showed significant LRRC4 methylation in nasopharyngeal carcinoma (Zhou et al., 2014). It is generally believed that DNA methylation can cause a commensurate reduction or disappearance of expression of the affected gene (Kim et al., 2015). Therefore, in this study, we used a greater number of nasopharyngeal tissue samples (than we did previously) to analyze the protein expression of $L R R C 4$ (nasopharyngitis and nasopharyngeal precancerous lesions served as the controls).

\section{MATERIAL AND METHODS}

\section{Patients and tissue samples}

The participants were outpatients of Zhongshan Hospital of Chinese Medicine in Guangdong Province; there were 50 cases [36.6 \pm 12.9 years old (mean \pm standard deviation)] of nasopharyngeal carcinoma, including 31 men and 19 women. The histological types were as follows: 38 cases of nonkeratinizing carcinoma, 8 cases of undifferentiated cancer, 4 cases of nonkeratinizing cancer mixed with undifferentiated cancer; the cases had the following clinical stages: 19 cases of stage I, 20 cases of stage II, 9 cases of stage III, and 2 cases of stage IV nasopharyngeal carcinoma. There were 28 cases ( $35.3 \pm 10.7$ years of age) of nasopharyngitis, including 16 men and 12 women. There were 22 cases ( $36.3 \pm 11.7$ years of age) of nasopharyngeal precancerous lesions, that included 12 men and 10 women.

Nasopharyngeal carcinoma was diagnosed using the conventional method of paraffinembedded slices; clinical staging of nasopharyngeal carcinoma was based on the clinical staging criteria proposed in 2008 (China Working Committee on Nasopharyngeal Carcinoma Clinical Staging, 2009) and involved conventional endoscopy of the nasopharynx, ultrasonography of the neck, systemic b-ultrasound, and nasopharyngeal computed tomography or magnetic resonance imaging to analyze the proliferation and metastasis of nasopharyngeal carcinoma and to identify the stages. Pathological classification of nasopharyngeal carcinoma was based on the criteria of "upper 
respiratory tract and ear histological classification" (Shanmugaratnam et al., 1991) proposed by the World Health Organization in 1991. Diagnosis of nasopharyngeal precancerous lesions involved the standards proposed by the Cancer Institute of Guangdong Zhongshan University (Zong et al., 2001), i.e., atypical squamous metaplasia or dysplasia in the nasopharyngeal epithelium was defined as a nasopharyngeal precancerous lesion. We used the following criteria for atypical cells: a significantly increased basal cell layer, varied cell sizes, poor differentiation, an aberrantly large nucleus, a prominent nucleolus, common karyokinesis, an increased ratio of the nucleus to the cytoplasm, disturbances or disappearance of cell polarity.

\section{Reagents and equipment}

Arabbit anti-human LRRC4 monoclonal antibody was purchased from Beijing Pherobio Technology Co., Ltd. (catalog No. WA-0821R); the Timely MaxVision Immunohistochemistry Kit (mouse/rabbit, cat. No. KIT-5010/5020/5030) was purchased from Fuzhou MXB Co., Ltd. Antigen heat-repairing reagents were purchased from Beijing Bioss Co., Ltd. (cat. No. C-0017).

\section{Immunohistochemistry}

Samples with PBS instead of the primary antibody were used as a negative control, and a biopsy specimen known to be positive for nasopharyngeal carcinoma served as a positive control. The acidic repair method was used for antigen retrieval: boiling in a sealed container in citrate buffer $\left(\mathrm{pH} 6.0,92^{\circ}-95^{\circ} \mathrm{C}\right.$ water bath) for $15 \mathrm{~min}$, with spontaneous cooling down to room temperature. After that, the samples were incubated with goat serum at $37^{\circ} \mathrm{C}$ for $20 \mathrm{~min}$ in a sealed container and then were incubated with the primary antibody at $4^{\circ} \mathrm{C}$ overnight. The staining steps were strictly in accordance with the instructions of the immunohistochemistry kit.

\section{Immunohistochemical criteria and statistical methods}

The cells with light-yellow-to-brown particles in the cytoplasm or nucleus were defined as test-positive cells. Under an optical microscope with 400X magnification, 5 visual fields were randomly selected in each slice for analysis of the cells, and the percentage of test-positive cells in each visual field was calculated; disputed results were settled by a simultaneous reading by 3 people and the majority vote. The calculation method was as follows (Wang et al., 2013): the total numbers of test-positive cells and test-negative cells in 5 visual fields were recorded; then we used the formula: the test-positive rate $=$ the number of test-positive cells $\times 100 \% /$ (the number of test-negative cells + the number of test-positive cells). At the same time, the intensity of testpositive staining of each slice was scored: no staining meant 0 points, light yellow staining meant 1 point, deep yellow staining meant 2 points, and brown staining denoted 3 points. We calculated the test-positive expression value as the test-positive rate multiplied by the staining intensity score. The grading criteria were as follows: when the test-positive expression was $<0.5$ and there was no protein expression, we recorded this result as grade 1; $0.5 \leq$ the test-positive expression $<1$ with weak protein expression: grade $2 ; 1 \leq$ the test-positive expression $<1.5$ with moderate protein expression: grade 3 ; the test-positive expression $\geq 1.5$ with strong protein expression: grade 4 .

SPSS 19.0 software was used for data processing. Grading was performed on the basis of the test-positive expression value; a nonparametric test (the Kruskal-Wallis test of $\mathrm{K}$ independent 
samples) was used for statistical analysis; pairwise comparisons were made using the Bonferroni method; differences with $\mathrm{P}<0.05$ were considered statistically significant.

\section{RESULTS}

\section{LRRC4 expression in nasopharyngeal carcinoma, nasopharyngeal precancerous lesions, and nasopharyngitis}

LRRC4 protein expression was mainly concentrated in the cytoplasm and was also detected in the nucleus; the expression of this protein manifested itself as yellow staining in our immunohistochemical assay; depending on the protein expression levels, the staining appeared as pale yellow, dark yellow, or brown (Figure 1).

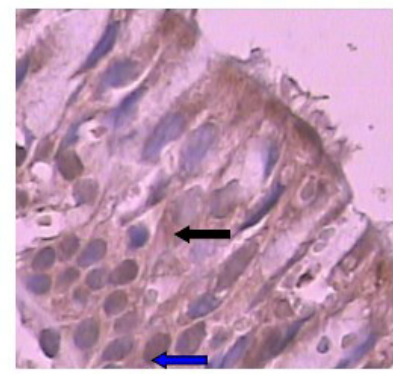

nasopharyngitis

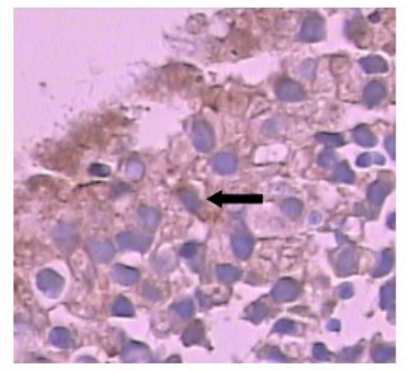

nasopharyngeal precancerosis

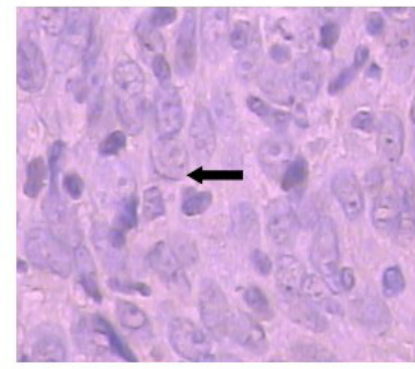

nasopharyngeal
carcinoma

Figure 1. LRRC4 protein expression innasopharyngitis, nasopharyngeal precancerous lesions, and nasopharyngeal carcinoma (immunohistochemical staining and 400X magnification). The black arrows indicate test-positive cytoplasm (grade 3), arrows show test-positive cytoplasm (grade 2), and arrows indicate test-negative cytoplasm. The blue arrow indicates test-positive staining of a nucleus.

LRRC4 showed strong expression in the epithelial tissues of patients with chronic nasopharyngitis and patients with nasopharyngeal precancerous lesions (a wide range and a high level of expression); these results appeared as brown staining, and the test-positive rates were 82.1 and $81.8 \%$ respectively. In contrast, LRRC4 was weakly expressed in nasopharyngeal carcinoma tissue, with a test-positive rate of $10.0 \%$; the expression levels of LRRC4 in oncological and nononcological nasopharyngeal diseases are shown in Table 1.

Table 1. Expression of the LRRC4 protein in nasopharyngeal lesions before nasopharyngeal cancer and in chronic nasopharyngitis.

\begin{tabular}{lcccrr}
\hline & $N$ & \multicolumn{3}{c}{ Detection of LRRC4 protein (number of cases) } \\
\cline { 3 - 5 } & & Level 1 & Level 2 & Level 3 & Level 4 \\
\hline Nasopharyngeal carcinoma & 50 & 44 & 5 & 1 & 0 \\
Nasopharyngeal precancerous lesions & 22 & 6 & 6 & 10 & 0 \\
Nasopharyngitis & 28 & 13 & 7 & 7 & 1 \\
\hline
\end{tabular}

Chi-square $=30.546, \mathrm{P}<0.0001$. 
LRRC4 expression was almost absent in nasopharyngeal carcinoma and was mainly detected in chronic nasopharyngitis and nasopharyngeal precancerous lesions. Pairwise comparisons showed that there were significant differences in LRRC4 expression between nasopharyngeal carcinoma and nasopharyngitis and between nasopharyngeal carcinoma and nasopharyngeal precancerous lesions, but there was no significant difference between nasopharyngitis and nasopharyngeal precancerous lesions.

\section{LRRC4 protein expression at different clinical stages of nasopharyngeal carcinoma}

These data are shown in Table 2. There was no significant difference in the expression of LRRC4 among different clinical stages.

Table 2. Expression of the LRRC4protein at different clinical stages of nasopharyngeal carcinoma (NPC).

\begin{tabular}{lcccc}
\hline & Number of cases & \multicolumn{2}{c}{ LRRC4 protein expression (number of cases) } \\
\cline { 3 - 5 } & & Level 1 & Level 2 \\
\hline Stage I NPC & 19 & 18 & 1 & Level 3 \\
Stage II NPC & 20 & 17 & 2 & 0 \\
Stage III NPC & 9 & 7 & 2 & 0 \\
Stage IV NPC & 2 & 2 & 0 & 0 \\
\hline
\end{tabular}

Chi-square $=2.089, \mathrm{P}=0.554(>0.05)$. The protein expression levels are listed from the weakest (Level 1$)$ to the strongest (Level 3).

\section{LRRC4 protein expression in different pathological types of nasopharyngeal carcinoma}

These data are shown in Table 3. There was no significant difference in the expression of LRRC4 among different pathological types of nasopharyngeal carcinoma.

Table 3. Expression of the LRRC4 protein in different histopathological grades of nasopharyngeal carcinoma.

\begin{tabular}{|c|c|c|c|c|}
\hline & \multirow[t]{2}{*}{ Number of cases } & \multicolumn{3}{|c|}{ LRRC4 protein expression (number of cases) } \\
\hline & & Level 1 & Level 2 & Level 3 \\
\hline Differentiated nonkeratinizing carcinoma & 38 & 35 & 2 & 1 \\
\hline $\begin{array}{l}\text { Mixed cancer: differentiated nonkeratinizing } \\
\text { carcinoma and undifferentiated carcinoma }\end{array}$ & 4 & 4 & 0 & 0 \\
\hline Undifferentiated carcinoma & 8 & 5 & 3 & 0 \\
\hline
\end{tabular}

\section{Analysis of cases of aberrant expressionof the LRRC4 protein}

In the nasopharyngitis-associated tissue samples, the LRRC4 protein was highly expressed; in the 5 cases without the LRRC4 protein expression, there was 1 case diagnosed as nasopharyngeal carcinoma after 6 months, 1 patient from a family with many cases of nasopharyngeal carcinoma, and 2 cases with nasopharyngeal precancerous lesions.

The LRRC4 protein was weakly expressed in nasopharyngeal carcinoma; even in the 5 cases of test-positive expression, the immunohistochemical staining was weak: pale yellow. 


\section{DISCUSSION}

The LRR domain was first discovered in human serum a2-glycoprotein in 1985 by Takahashi et al. (1985) and was named after the regular repeats of leucine residues in its structure. The $L R R C 4$ gene is located in the chromosomal region 7q32.1; the full length of the cDNA is $1962 \mathrm{bp}$. The Entrez protein accession No. is NP_071426, and the GenBank accession No. is AFI96976. The gene contains multiple LRR sequences that have structural composition similar to that of the LRR superfamily members in humans, yeast, mice, rats, cattle, and zebrafish; therefore, the gene is considered a new member of the LRR superfamily with a highly conserved sequence; it was named LRRC4 (the name was approved by the International Human Genome Nomenclature Committee). The LRRC4 gene encodes an enzyme (a secretory protein or membrane-bound protein) catalyzing dephosphorylation of proteins and playing an important role in the regulation of protein dephosphorylation that is mainly related to the development and function of the nervous system.

$L R R C 4$ expression is relatively specific to the brain and is absent in the heart, liver, lung, stomach, esophagus, small intestine, large intestine, kidney, testis, thymus, pancreas, adrenal gland, skeletal muscle, bladder, skin, placenta, uterus, and leukocytes in peripheral blood. LRRC4 expression is weak or absent in a variety of primary brain tumor tissues (60 known cases); the incidence of inactivation or downregulation is $87.5 \%$ in glioma (21/24), $80.9 \%$ in meningioma $(17 / 21)$, and $85.2 \%$ in pituitary and other brain tumors. A study on the expression in different grades of brain glioma showed that $L R R C 4$ is expressed more strongly in grade I, and there are no significant differences with healthy brain tissue; nevertheless, the expression is significantly reduced or absent in grade II and absent in grades III and IV. Therefore, LRRC4 seems to be involved in maintaining the normal function of the nervous system, and its expression correlates negatively with the progression of gliomas (Wang et al., 2002; Wu et al., 2006).

The results of our study show that the LRRC4 protein is barely expressed if at all in nasopharyngeal carcinoma and shows high expression levels in chronic nasopharyngitis and nasopharyngeal precancerous lesions. The difference is statistically significant.

Pairwise comparison showed that there is no significant difference in LRRC4 expression between chronic nasopharyngitis and nasopharyngeal precancerous lesions, but there are statistically significant differences between each of them and nasopharyngeal carcinoma, indicating that disappearance of LRRC4 expression occurs only in nasopharyngeal carcinoma. There are no significant differences in the LRRC4 protein expression among different pathological types and different clinical stages of nasopharyngeal carcinoma according to our results, even though LRRC4 expression is mostly absent in medium-grade and advanced glioma (Wang et al., 2002). These findings suggest that low expression of LRRC4 is one of the characteristics of nasopharyngeal cancer, and it does not significantly correlate with progression of nasopharyngeal carcinoma.

Tumorigenesis and tumor progression are usually driven by interaction of genetic and epigenetic changes. Carcinogenesis is a pathological process involving multiple genes and multistage accumulation of mutations. Inactivation of tumor suppressor genes is one of the key factors. There are many mechanisms of the loss of function of a tumor suppressor gene (Mobley et al., 2015). The DNA methylation is the 3rd most common mechanism after gene deletion and a mutation that inactivates a tumor suppressor gene; in some cases, DNA methylationis the only mechanism of the inactivation (Herman et al., 2003). Our previous study (Zhou et al., 2013) showed methylation status before and after the development of a carcinoma in 3 cases of nasopharyngeal precancerous lesions; we found that there is substantial methylation of the 
$L R R C 4$ gene promoter region after the development of nasopharyngeal carcinoma, and there is a large number of methylation peaks in the sequence. Studieson brain glioma also showed that aberrant methylation of the $L R R C 4$ promoter is an important mechanism behind the lack of $L R R C 4$ expression in glioma cells (Zhang et al., 2009; Tang et al., 2012). The present result suggests that the substantial methylation of the upstream region of the $L R R C 4$ promoter abrogates the $L R R C 4$ expression in nasopharyngeal carcinoma. This mechanism may be important for the development of nasopharyngeal carcinoma.

\section{Conflicts of interest}

The authors declare no conflict of interest.

\section{ACKNOWLEDGMENTS}

\#81072847.

\section{REFERENCES}

China Working Committee on NPC Clinical Staging (2009). Nasopharyngeal 92 installments revision report. Chin. J. Radiat. Oncol. 18: 2-6.

Herman JG and Baylin SB (2003). Gene silencing in cancer in association with promoter hypermethylation. N. Engl. J. Med. 349: 2042-2054.

Kim JD, Lee A, Choi J, Park Y, et al. (2015). Epigenetic modulation as a therapeutic approach for pulmonary arterial hypertension. Exp. Mol. Med. 47: e175.

Mobley BC, Kwon M, Kraemer BR, Hickman FE, et al. (2015). Expression of MYCN in multipotent sympathoadrenal progenitors induces proliferation and neural differentiation, but Is not sufficient for tumorigenesis. PLoS One 10: e0133897.

Shanmugaratnam K, Sobin LH, Barnes L, Smith K, et al. (1991). World Health Organization histological classification of tumours. Histological typing of tumours of the upper respiratory tract and ear. 2nd edn. Springer-Verlag, New York.

Takahashi N, Takahashi Y and Putnam FW (1985). Periodicity of leucine and tandem repetition of a 24-amino acid segment in the primary structure of leucine-rich a2-glycoprotein of human serum. Proc. Natl. Acad. Sci. U. S. A. 82: 1906-1910.

Tang H, Wang Z, Liu X, Liu Q, et al. (2012) LRRC4 inhibits glioma cell growth and invasion through a miR-185-dependent pathway. Curr. Cancer Drug Targets 12: 1032-1042.

Wang JR, Qian J, Dong L, Li XL, et al. (2002). Identification of LRRC4, a novel member of leucine-rich repeat (LRR) Superfamily, and its expression analysis in brain tumor. Prog. Biochem. Biophys. 29: 233-239.

Wang Y, Wang SQ, Zhou XJ, Wang J, et al. (2013). The expressive activities of CyclinD1 and Skp-2 in tissues of nasopharyngeal carcinoma and nasopharyngeal precancerous lesion and their implications in carcinogenesis. Chin. J. Otorhlnteg. Med. 21: 171-173.

Wu M, Huang C, Gan K, Huang H, et al. (2006). LRRC4, a putative tumor suppressor gene, requires a functional leucine-rich repeat cassette domain to inhibit proliferation of glioma cells in vitro by modulating the extracellular signal-regulated kinase/protein kinase B/nuclear factor-\{kappa\}B pathway. Mol. Biol. Cell 17: 3534-3542.

Zhang ZP, Wu MH, Tang HL, Wang R, et al. (2009). Effects of 5-aza-2'-deoxycitydine on proliferation of glioma cell lines and abnormal methylation of LRRC4 gene. Prog. Biochem. Biophys. 36: 904-909.

Zhou XJ, Chen JH, Wang SZ, Peng W, et al. (2013). Study on the correlation of nasopharyngeal epithelium canceration with DNA methylation features. Chin. J. Otorhin. Integr. Med. 21: 22-26.

Zhou XJ, Chen JH, Wang SZ, Wang S, et al. (2014). Study on methylation of ADAM23, MIMT1, FAM150B, GRIN2A, LRRC4 before and after nasopharyngeal carcinoma canceration. J. Chongqing Med. Univ. 39: 341-344.

Zong YS, Wu QL, Liang XM, Liang YJ, et al. (2001). The study of histological type and precancerous lesions of nasopharyngeal carcinoma. Ai Zheng 20: 117-127. 